\title{
Coefficient Estimates of Some Classes of Univalent Functions using Subordination Principle
}

\author{
Olubunmi A. Fadipe-Joseph ${ }^{1}$, E. A. Aina ${ }^{2}$ and E. O. Titiloye ${ }^{3}$ \\ ${ }^{1}$ Department of Mathematics, University of Ilorin, P. M. B. 1515, Ilorin, Nigeria \\ e-mail: famelov@unilorin.edu.ng; famelov@gmail.com \\ ${ }^{2}$ Department of Mathematics, University of Ilorin, P. M. B. 1515, Ilorin, Nigeria \\ e-mail: ebenezer.a.aina@gmail.com \\ ${ }^{3}$ Department of Mathematics, University of Ilorin, P. M. B. 1515, Ilorin, Nigeria \\ e-mail: eotitiloye@gmail.com
}

\begin{abstract}
In this work, two classes $T(b, \lambda)$ and $V(b, \lambda)$ were defined. Coefficient bounds, FeketeSzegö functional and Hankel determinants for the classes were obtained. The results obtained generalized some earlier ones.
\end{abstract}

\section{Introduction and Preliminaries}

Let $A$ be the class of analytic functions $f(z)$ of the form:

$$
f(z)=z+\sum_{n=2}^{\infty} a_{n} z^{n}
$$

in $\mathbb{U}=\{z \in \mathbb{C}:|z|<1\}$. Furthermore, let $S$ represent the family of all functions in $A$ which are univalent in $\mathbb{U}$.

Received: February 27, 2020; Accepted: March 28, 2020

2010 Mathematics Subject Classification: Primary 30C45; Secondary 30C80.

Keywords and phrases: subordination principle, univalent functions, modified sigmoid function, FeketeSzegö functional, Hankel determinant.

Copyright $\odot 2020$ Olubunmi A. Fadipe-Joseph et al. This is an open access article distributed under the Creative Commons Attribution License, which permits unrestricted use, distribution, and reproduction in any medium, provided the original work is properly cited. 
Similarly, the class $P$ of all analytic functions $\phi$ with positive real part in $\mathbb{D}$ such that $\phi(0)=1$ had been used by many authors. For functions in the class $P$ expressed in the form

$$
\phi(z)=1+\sum_{n=1}^{\infty} c_{n} z^{n}
$$

sharp bound $\left|c_{n}\right| \leq 2, n=1,2, \ldots$ exist.

Using subordination principle defined in literature such as Miller and Mocanu [6], some results have been established by many authors.

Kuroki and Owa [4] obtained the conditions necessary and sufficient for any function $f(z) \in A$ to satisfy the subordination

$$
\frac{z f^{\prime}(z)}{f(z)} \prec 1+\frac{(\beta-\alpha)}{\pi} i \log \left(\frac{1-e^{i \frac{\pi(1-\alpha)}{\beta-\alpha}} z}{1-e^{-i \frac{\pi(1-\alpha)}{\beta-\alpha}} z}\right),
$$

and remarked as follows:

Let $S_{\alpha, \beta}(z): \mathbb{U} \rightarrow \mathbb{C}$

$$
S_{\alpha, \beta}(z)=1+\frac{(\beta-\alpha)}{\pi} i \log \left(\frac{1-e^{i \frac{\pi(1-\alpha)}{\beta-\alpha} z}}{1-e^{-i \frac{\pi(1-\alpha)}{\beta-\alpha}} z}\right)=1+\sum_{n=1}^{\infty} B_{n} z^{n},
$$

where

$$
B_{n}=\frac{2(\beta-\alpha)}{n \pi} \sin \left(\frac{n \pi(1-\alpha)}{\beta-\alpha}\right) \quad \alpha<1, \beta>1
$$

(For details, see Remark 1.3 in [4]).

Fadipe-Joseph et al. [3] defined the modified sigmoid function

$$
G(z)=\frac{2}{1+e^{-z}}=1+\left(\sum_{m=1}^{\infty} \frac{(-1)^{m}}{2^{m}}\left[\sum_{n=1}^{\infty} \frac{(-1)^{n}}{n !} z^{n}\right]^{m}\right) \in P .
$$


Altinkaya and Yalçin [1] gave the coefficient estimate of a class of bi-univalent functions using the Schwarz function

$$
u(z)=\frac{p(z)-1}{p(z)+1}=\frac{1}{2} p_{1} z+\frac{1}{2}\left(p_{2}-\frac{1}{2} p_{1}^{2}\right) z^{2}+\ldots
$$

Malik et al. [5] also used Schwarz function in obtaining the coefficient inequality of functions in the class they defined using subordination principle.

Hence, subordination principle was used to establish the coefficient bounds of some classes of univalent functions in this paper.

\section{Main Results}

Definition 2.1. A function $f \in A$ is said to be in the class $T(b, \lambda) ; 0 \neq b \in \mathbb{C}$, $\lambda \geq 1$ if the following subordination holds

$$
1+\frac{1}{b}\left(\frac{z\left(f^{\prime}(z)\right)^{\lambda}}{f(z)}-1\right) \prec S_{\alpha, \beta}(z) .
$$

Theorem 2.1. Let $f \in T(b, \lambda)$. Then

$$
\begin{aligned}
\left|a_{2}\right| \leq & \frac{2|b|(\beta-\alpha)}{(2 \lambda-1) \pi} \sin \left(\frac{\pi(1-\alpha)}{\beta-\alpha}\right) \\
\left|a_{3}\right| \leq & \frac{2|b|(\beta-\alpha)}{(3 \lambda-1) \pi} \sin \left(\frac{\pi(1-\alpha)}{\beta-\alpha}\right)\left|\cos \left(\frac{\pi(1-\alpha)}{\beta-\alpha}\right)+\frac{2|b|(\beta-\alpha)\left|4 \lambda-2 \lambda^{2}-1\right|}{\pi(2 \lambda-1)^{2}} \sin \left(\frac{\pi(1-\alpha)}{\beta-\alpha}\right)\right| \\
\left|a_{4}\right| \leq & \frac{2|b|(\beta-\alpha)}{(4 \lambda-1) \pi}\left(4+4\left|\cos \left(\frac{\pi(1-\alpha)}{\beta-\alpha}\right)\right|+\left|\cos ^{2}\left(\frac{\pi(1-\alpha)}{\beta-\alpha}\right)-\frac{1}{3} \sin ^{2}\left(\frac{\pi(1-\alpha)}{\beta-\alpha}\right)\right|\right. \\
& +\frac{\left|11 \lambda-6 \lambda^{2}-2\right||b|(\beta-\alpha)}{(2 \lambda-1)(3 \lambda-1) \pi} \sin \left(\frac{\pi(1-\alpha)}{\beta-\alpha}\right) \\
& 2\left(1+\left|\cos \frac{\pi(1-\alpha)}{\beta-\alpha}-1\right|+\frac{2|b|(\beta-\alpha)\left|4 \lambda-2 \lambda^{2}-1\right|}{\pi(2 \lambda-1)^{2}} \sin \frac{\pi(1-\alpha)}{\beta-\alpha}\right) \\
& \left.+\frac{4\left|6 \lambda^{2}-\frac{4}{3} \lambda^{3}-\frac{20}{3} \lambda+1\right| \cdot|b|^{2}(\beta-\alpha)^{2}}{(2 \lambda-1)^{3} \pi^{2}} \sin ^{2} \frac{\pi(1-\alpha)}{\beta-\alpha}\right) .
\end{aligned}
$$


Proof. Suppose $f \in T(b, \lambda)$, then by definition

$$
1+\frac{1}{b}\left(\frac{z\left(f^{\prime}(z)\right)^{\lambda}}{f(z)}-1\right) \prec S_{\alpha, \beta}(z)
$$

Consider

$$
p(z)=1+p_{1} z+p_{2} z^{2}+p_{3} z^{3}+\ldots \in P .
$$

Let

$$
w(z)=\frac{p(z)-1}{p(z)+1}=\frac{p_{1}}{2} z+\frac{1}{2}\left(p_{2}-\frac{p_{1}^{2}}{2}\right) z^{2}+\frac{1}{2}\left(p_{3}-p_{1} p_{2}+\frac{p_{1}^{3}}{4}\right) z^{3}+\ldots
$$

Therefore,

$$
\begin{aligned}
1+ & \frac{1}{b}\left(\frac{z\left(f^{\prime}(z)\right)^{\lambda}}{f(z)}-1\right)=S_{\alpha, \beta}(w(z)) \\
& 1+\frac{2 \lambda-1}{b} a_{2} z+\frac{\left((3 \lambda-1) a_{3}+\left(2 \lambda^{2}-4 \lambda+1\right) a_{2}^{2}\right) z^{2}}{b} \\
& \frac{\left((4 \lambda-1) a_{4}+\left(6 \lambda^{2}-11 \lambda+2\right) a_{2} a_{3}+\left(\frac{4}{3} \lambda^{3}-6 \lambda^{2}+\frac{20}{3} \lambda-1\right) a_{2}^{3}\right) z^{3}}{b}+\ldots \\
= & 1+\frac{B_{1} p_{1}}{2} z+\left(\frac{B_{1} p_{2}}{2}+\frac{\left(B_{2}-B_{1}\right) p_{1}^{2}}{4}\right) z^{2} \\
& +\left(\frac{B_{1} p_{3}}{2}+\frac{\left(B_{2}-B_{1}\right) p_{1} p_{2}}{2}+\frac{\left(B_{1}-2 B_{2}+B_{3}\right) p_{1}^{3}}{8}\right) z^{3}+\ldots
\end{aligned}
$$

Comparing coefficients of $z, z^{2}$ and $z^{3}$;

$$
\begin{gathered}
a_{2}=\frac{b p_{1}}{2(2 \lambda-1)}\left(\frac{2(\beta-\alpha)}{\pi} \sin \left(\frac{\pi(1-\alpha)}{\beta-\alpha}\right)\right) \\
\left|a_{2}\right| \leq \frac{2|b|(\beta-\alpha)}{(2 \lambda-1) \pi} \sin \left(\frac{\pi(1-\alpha)}{\beta-\alpha}\right) .
\end{gathered}
$$


Similarly,

$$
\begin{aligned}
& a_{3}=\frac{b(\beta-\alpha)}{(3 \lambda-1) \pi} \sin \left(\frac{\pi(1-\alpha)}{\beta-\alpha}\right)\left(p_{2}+\frac{p_{1}^{2}}{2} \cos \left(\frac{\pi(1-\alpha)}{\beta-\alpha}\right)-\frac{p_{1}^{2}}{2}\right. \\
& \left.+\frac{b p_{1}^{2}(\beta-\alpha)\left(4 \lambda-2 \lambda^{2}-1\right)}{\pi(2 \lambda-1)^{2}} \sin \left(\frac{\pi(1-\alpha)}{\beta-\alpha}\right)\right) \\
& \left|a_{3}\right| \leq \frac{2|b|(\beta-\alpha)}{(3 \lambda-1) \pi} \sin \left(\frac{\pi(1-\alpha)}{\beta-\alpha}\right) \\
& \left|\cos \left(\frac{\pi(1-\alpha)}{\beta-\alpha}\right)+\frac{\left.2|b|(\beta-\alpha) \mid 4 \lambda-2 \lambda^{2}-1\right]}{\pi(2 \lambda-1)^{2}} \sin \left(\frac{\pi(1-\alpha)}{\beta-\alpha}\right)\right|, \\
& a_{4}=\frac{b(\beta-\alpha)}{(4 \lambda-1) \pi} \sin \left(\frac{\pi(1-\alpha)}{\beta-\alpha}\right)\left(\left(p_{3}-p_{1} p_{2}+\frac{p_{1}^{3}}{4}\right)+\left(p_{1} p_{2}-\frac{p_{1}^{3}}{2}\right) \cos \left(\frac{\pi(1-\alpha)}{\beta-\alpha}\right)\right. \\
& +\left(\frac{p_{1}^{3}}{8}\right)\left(\frac{2}{3}\left(3 \cos ^{2}\left(\frac{\pi(1-\alpha)}{\beta-\alpha}\right)-\sin ^{2}\left(\frac{\pi(1-\alpha)}{\beta-\alpha}\right)\right)\right) \\
& +\frac{11 \lambda-6 \lambda^{2}-2}{(2 \lambda-1)(3 \lambda-1)}\left(\frac{b p_{1}(\beta-\alpha)}{\pi} \sin \left(\frac{\pi(1-\alpha)}{\beta-\alpha}\right)\right) \\
& \left(p_{2}+\frac{p_{1}^{2}}{2} \cos \left(\frac{\pi(1-\alpha)}{\beta-\alpha}\right)-\frac{p_{1}^{2}}{2}+\frac{b p_{1}^{2}(\beta-\alpha)\left(4 \lambda-2 \lambda^{2}-1\right)}{\pi(2 \lambda-1)^{2}} \sin \left(\frac{\pi(1-\alpha)}{\beta-\alpha}\right)\right) \\
& \left.+\frac{\left(\frac{4}{3} \lambda^{3}-6 \lambda^{2}+\frac{20}{3} \lambda-1\right) b^{2} p_{1}^{3}(\beta-\alpha)^{2}}{3 \pi^{2}(2 \lambda-1)^{3}} \sin ^{2}\left(\frac{\pi(1-\alpha)}{\beta-\alpha}\right)\right)
\end{aligned}
$$$$
\left|a_{4}\right| \leq \frac{2|b|(\beta-\alpha)}{(4 \lambda-1) \pi}\left(4+4\left|\cos \left(\frac{\pi(1-\alpha)}{\beta-\alpha}\right)\right|\left|\cos ^{2}\left(\frac{\pi(1-\alpha)}{\beta-\alpha}\right)-\frac{1}{3} \sin ^{2}\left(\frac{\pi(1-\alpha)}{\beta-\alpha}\right)\right|\right.
$$$$
+\frac{\left|11 \lambda-6 \lambda^{2}-2\right||b|(\beta-\alpha)}{(2 \lambda-1)(3 \lambda-1) \pi} \sin \left(\frac{\pi(1-\alpha)}{\beta-\alpha}\right)
$$ 


$$
\begin{aligned}
& 2\left(1+\left|\cos \frac{\pi(1-\alpha)}{\beta-\alpha}-1\right|+\frac{2|b|(\beta-\alpha)\left|4 \lambda-2 \lambda^{2}-1\right|}{\pi(2 \lambda-1)^{2}} \sin \frac{\pi(1-\alpha)}{\beta-\alpha}\right) \\
& \left.+\frac{4\left|6 \lambda^{2}-\frac{4}{3} \lambda^{3}-\frac{20}{3} \lambda+1\right| \cdot|b|^{2}(\beta-\alpha)^{2}}{(2 \lambda-1)^{3} \pi^{2}} \sin ^{2} \frac{\pi(1-\alpha)}{\beta-\alpha}\right) .
\end{aligned}
$$

Theorem 2.2. Let $f \in T(b, \lambda)$. Then

$$
\begin{aligned}
\left|a_{3}-\mu a_{2}^{2}\right| \leq & \frac{2|b|(\beta-\alpha)}{(3 \lambda-1) \pi} \sin \left(\frac{\pi(1-\alpha)}{\beta-\alpha}\right)\left(1+\left|\cos \left(\frac{\pi(1-\alpha)}{\beta-\alpha}\right)-1\right|\right. \\
& \left.+\frac{2\left|(4-3 \mu) \lambda-2 \lambda^{2}-(1-\mu)\right||b|(\beta-\alpha)}{\pi(2 \lambda-1)^{2}} \sin \left(\frac{\pi(1-\alpha)}{\beta-\alpha}\right)\right) .
\end{aligned}
$$

\section{Proof.}

$$
\begin{aligned}
a_{3}-\mu a_{2}^{2}= & \frac{b}{3 \lambda-1}\left(\frac{B_{1} p_{2}}{2}+\frac{\left(B_{2}-B_{1}\right) p_{1}^{2}}{4}+\frac{\left(4 \lambda-2 \lambda^{2}-1\right)}{b} a_{2}^{2}\right)-\mu a_{2}^{2} \\
= & \frac{b(\beta-\alpha)}{(3 \lambda-1) \pi} \sin \left(\frac{\pi(1-\alpha)}{\beta-\alpha}\right)\left(\left(p_{2}-\frac{p_{1}^{2}}{2}\right)+\frac{p_{1}^{2}}{2} \cos \left(\frac{\pi(1-\alpha)}{\beta-\alpha}\right)\right. \\
& \left.+\frac{(4-3 \mu) \lambda-2 \lambda^{2}-(1-\mu)}{\pi^{2}(2 \lambda-1)^{2}} b p_{1}^{2}(\beta-\alpha) \sin \left(\frac{\pi(1-\alpha)}{\beta-\alpha}\right)\right) \\
\left|a_{3}-\mu a_{2}^{2}\right| \leq & \left|\frac{b(\beta-\alpha)}{(3 \lambda-1) \pi} \sin \left(\frac{\pi(1-\alpha)}{\beta-\alpha}\right)\right|\left(\left|p_{2}-\frac{p_{1}^{2}}{2}+\frac{p_{1}^{2}}{2} \cos \left(\frac{\pi(1-\alpha)}{\beta-\alpha}\right)\right|\right. \\
& \left.+\left|\frac{(4-3 \mu) \lambda-2 \lambda^{2}-(1-\mu)}{\pi^{2}(2 \lambda-1)^{2}} b p_{1}^{2}(\beta-\alpha) \sin \left(\frac{\pi(1-\alpha)}{\beta-\alpha}\right)\right|\right) \\
\left|a_{3}-\mu a_{2}^{2}\right| \leq & \frac{2|b|(\beta-\alpha)}{(3 \lambda-1) \pi} \sin \left(\frac{\pi(1-\alpha)}{\beta-\alpha}\right)\left(1+\left|\cos \left(\frac{\pi(1-\alpha)}{\beta-\alpha}\right)-1\right|\right. \\
& \left.+\frac{2\left|(4-3 \mu) \lambda-2 \lambda^{2}-(1-\mu)\right||b|(\beta-\alpha)}{\pi(2 \lambda-1)^{2}} \sin \left(\frac{\pi(1-\alpha)}{\beta-\alpha}\right)\right)
\end{aligned}
$$


Theorem 2.3. Let $f \in T(b, \lambda)$. Then

$$
\begin{aligned}
\left|a_{4}-a_{2} a_{3}\right| \leq & \frac{2(\beta-\alpha)|b|}{(4 \lambda-1) \pi} \sin \frac{\pi(1-\alpha)}{\beta-\alpha}\left(1+2\left|\cos \frac{\pi(1-\alpha)}{\beta-\alpha}-1\right|\right. \\
& +\left|1-2 \cos \frac{\pi(1-\alpha)}{\beta-\alpha}+\cos ^{2} \frac{\pi(1-\alpha)}{\beta-\alpha}+\frac{1}{3} \sin ^{2} \frac{\pi(1-\alpha)}{\beta-\alpha}\right| \\
& +\frac{\left|7 \lambda-6 \lambda^{2}-1\right| \cdot|b|(\beta-\alpha)}{(2 \lambda-1)(3 \lambda-1) \pi}\left(1+\left|\cos \frac{\pi(1-\alpha)}{\beta-\alpha}-1\right|\right) \sin \frac{\pi(1-\alpha)}{\beta-\alpha} \\
& \left.\frac{4\left|\frac{34}{3} \lambda^{3}-4 \lambda^{4}-8 \lambda^{2}+\frac{5}{3} \lambda\right||b|^{2}(\beta-\alpha)^{2}}{(2 \lambda-1)^{3}(3 \lambda-1) \pi^{2}} \sin ^{2} \frac{\pi(1-\alpha)}{\beta-\alpha}\right) .
\end{aligned}
$$

\section{Proof.}

$$
\begin{aligned}
a_{4}-a_{2} a_{3}= & \frac{b B_{1} p_{3}}{2(4 \lambda-1)}+\frac{b\left(B_{2}-B_{1}\right) p_{1} p_{2}}{2(4 \lambda-1)} \\
& +\frac{b\left(B_{1}-2 B_{2}+B_{3}\right) p_{1}^{3}}{8(4 \lambda-1)}-\frac{\frac{4}{3} \lambda^{3}-6 \lambda^{2}+\frac{20}{3} \lambda-1}{4 \lambda-1} a_{2}^{3} \\
& -\left(\frac{6 \lambda^{2}-11 \lambda+2}{4 \lambda-1}+1\right) a_{2} a_{3} \\
= & \frac{b B_{1} p_{3}}{2(4 \lambda-1)}+\frac{b\left(B_{2}-B_{1}\right) p_{1} p_{2}}{2(4 \lambda-1)}+\frac{b\left(B_{1}-2 B_{2}+B_{3}\right) p_{1}^{3}}{8(4 \lambda-1)} \\
& -\frac{6 \lambda^{2}-7 \lambda+1}{4 \lambda-1}\left(\frac{\left(2 p_{2}-p_{1}^{2}\right) B_{1}+p_{1}^{2} B_{2}}{4(3 \lambda-1)}\right) a_{2} b \\
& -\frac{\frac{4}{3} \lambda^{3}-6 \lambda^{2}+\frac{20}{3} \lambda-1}{4 \lambda-1} a_{2}^{3}-\frac{2 \lambda^{2}-4 \lambda+1}{3 \lambda-1} a_{2}^{3} \\
= & \frac{\beta-\alpha}{\pi} \sin \frac{\pi(1-\alpha)}{\beta-\alpha}\left(\frac{b p_{3}}{(4 \lambda-1)}+\frac{b p_{1} p_{2}}{4 \lambda-1}\left(\cos \frac{\pi(1-\alpha)}{\beta-\alpha}-1\right)\right.
\end{aligned}
$$




$$
\begin{aligned}
& +\frac{b p_{1}^{3}}{4(4 \lambda-1)}\left(1-2 \cos \frac{\pi(1-\alpha)}{\beta-\alpha}+\cos ^{2} \frac{\pi(1-\alpha)}{\beta-\alpha}+\frac{1}{3} \sin ^{2} \frac{\pi(1-\alpha)}{\beta-\alpha}\right) \\
& +\frac{2\left(7 \lambda-6 \lambda^{2}-1\right)}{4 \lambda-1} \frac{b^{2} p_{1}(\beta-\alpha)}{(2 \lambda-1) \pi} \sin \frac{\pi(1-\alpha)}{\beta-\alpha}\left(\frac{2 p_{2}+p_{1}^{2}\left(\cos \frac{\pi(1-\alpha)}{\beta-\alpha}-1\right)}{4(3 \lambda-1)}\right) \\
& \left.-\frac{\frac{34}{3} \lambda^{3}-4 \lambda^{4}-8 \lambda^{2}+\frac{5}{3} \lambda}{(4 \lambda-1)(3 \lambda-1)} \frac{b^{3} p_{1}^{3}(\beta-\alpha)^{2}}{(2 \lambda-1)^{3} \pi^{2}} \sin ^{2} \frac{\pi(1-\alpha)}{\beta-\alpha}\right) \\
& \left|a_{4}-a_{2} a_{3}\right| \leq \frac{2(\beta-\alpha)|b|}{(4 \lambda-1) \pi} \sin \frac{\pi(1-\alpha)}{\beta-\alpha}\left(1+2\left|\cos \left(\frac{\pi(1-\alpha)}{\beta-\alpha}\right)-1\right|\right. \\
& +\left|1-2 \cos \frac{\pi(1-\alpha)}{\beta-\alpha}+\cos ^{2} \frac{\pi(1-\alpha)}{\beta-\alpha}+\frac{1}{3} \sin ^{2} \frac{\pi(1-\alpha)}{\beta-\alpha}\right| \\
& +\frac{\left|7 \lambda-6 \lambda^{2}-1\right| \cdot|b|(\beta-\alpha)}{(2 \lambda-1)(3 \lambda-1) \pi}\left(1+\left|\cos \frac{\pi(1-\alpha)}{\beta-\alpha}-1\right|\right) \sin \frac{\pi(1-\alpha)}{\beta-\alpha} \\
& \left.\frac{4\left|\frac{34}{3} \lambda^{3}-4 \lambda^{4}-8 \lambda^{2}+\frac{5}{3} \lambda\right||b|^{2}(\beta-\alpha)^{2}}{(2 \lambda-1)^{3}(3 \lambda-1) \pi^{2}} \sin ^{2} \frac{\pi(1-\alpha)}{\beta-\alpha}\right) .
\end{aligned}
$$

Remark 2.1. Let $f(z) \in T(1,1)$. Then $\left|a_{2}\right|$ and $\left|a_{3}\right|$ agree with the bounds in Kuroki and Owa [4].

Definition 2.2. A function $f \in A$ is said to be in the class $V(b, \lambda) ; 0 \neq b \in \mathbb{C}$, $\lambda \geq 1$ if the following subordination holds.

$$
1+\frac{1}{b}\left(\frac{z\left(f^{\prime}(z)\right)^{\lambda}}{f(z)}-1\right) \prec G(z)=1+\frac{1}{2} z-\frac{1}{24} z^{3}+\frac{1}{240} z^{5}-\cdots
$$

Theorem 2.4. Let $f \in V(b, \lambda)$. Then

$$
\left|a_{2}\right| \leq \frac{|b|}{2(2 \lambda-1)}
$$




$$
\begin{aligned}
\left|a_{3}\right| \leq & \frac{|b|}{4(3 \lambda-1)}\left(2+\frac{|b| \cdot\left|4 \lambda-2 \lambda^{2}-1\right|}{(2 \lambda-1)^{2}}\right) \\
\left|a_{4}\right| \leq & \frac{|b|}{8(4 \lambda-1)}\left(31 \frac{2}{3}+\frac{|b| \cdot\left|11 \lambda-6 \lambda^{2}-2\right|}{(2 \lambda-1)(3 \lambda-1)}\left(2+\frac{|b| \cdot\left|4 \lambda-2 \lambda^{2}-1\right|}{(2 \lambda-1)^{2}}\right)\right. \\
& \left.+\frac{|b|^{2}\left|6 \lambda^{2}-\frac{4}{3} \lambda^{3}-\frac{20}{3} \lambda+1\right|}{(2 \lambda-1)^{3}}\right) .
\end{aligned}
$$

\section{Proof.}

$$
\begin{aligned}
& 1+\frac{1}{b}\left(\frac{z\left(f^{\prime}(z)\right)^{\lambda}}{f(z)}-1\right)=G(w(z)) \\
& 1+\frac{2 \lambda-1}{b} a_{2} z+\frac{\left((3 \lambda-1) a_{3}+\left(2 \lambda^{2}-4 \lambda+1\right) a_{2}^{2}\right) z^{2}}{b} \\
& +\frac{\left((4 \lambda-1) a_{4}+\left(6 \lambda^{2}-11 \lambda+2\right) a_{2} a_{3}+\left(\frac{4}{3} \lambda^{3}-6 \lambda^{2}+\frac{20}{3} \lambda-1\right) a_{2}^{3}\right) z^{3}}{b}+\ldots \\
& =1+\frac{p_{1}}{4} z+\frac{1}{8}\left(2 p_{2}-p_{1}^{2}\right) z^{2}+\frac{1}{8}\left(4 p_{3}-4 p_{1} p_{2}+\frac{23}{24} p_{1}^{3}\right) z^{3}+\ldots
\end{aligned}
$$

Comparing coefficient of $z, z^{2}$ and $z^{3}$;

$$
\begin{aligned}
& a_{2}=\frac{b p_{1}}{4(2 \lambda-1)} \\
& \left|a_{2}\right| \leq \frac{|b|}{2(2 \lambda-1)} \\
& a_{3}=\frac{b}{8(3 \lambda-1)}\left(\left(2 p_{2}-p_{1}^{2}\right)+\frac{b p_{1}^{2}\left(4 \lambda-2 \lambda^{2}-1\right)}{2(2 \lambda-1)^{2}}\right) \\
& \left|a_{3}\right| \leq \frac{|b|}{4(3 \lambda-1)}\left(2+\frac{|b| \cdot\left|4 \lambda-2 \lambda^{2}-1\right|}{(2 \lambda-1)^{2}}\right)
\end{aligned}
$$




$$
\begin{aligned}
a_{4}= & \frac{1}{(4 \lambda-1)}\left(\frac{b}{8}\left(4 p_{3}-4 p_{1} p_{2}+\frac{23}{24} p_{1}^{3}\right)\right. \\
& +\left(11 \lambda-6 \lambda^{2}-2\right)\left(\frac{b p_{1}}{4(2 \lambda-1)}\right)\left(\frac{1}{8(3 \lambda-1)}\left(b\left(2 p_{2}-p_{1}^{2}\right)+\frac{b^{2} p_{1}^{2}\left(4 \lambda-2 \lambda^{2}-1\right)}{2(2 \lambda-1)^{2}}\right)\right) \\
& \left.+\left(6 \lambda^{2}-\frac{4}{3} \lambda^{3}-\frac{20}{3} \lambda+1\right)\left(\frac{b^{3} p_{1}^{3}}{64(2 \lambda-1)^{3}}\right)\right) \\
\left|a_{4}\right| \leq & \frac{|b|}{8(4 \lambda-1)}\left(31 \frac{2}{3}+\frac{|b| \cdot\left|11 \lambda-6 \lambda^{2}-2\right|}{(2 \lambda-1)(3 \lambda-1)}\left(2+\frac{|b| \cdot\left|4 \lambda-2 \lambda^{2}-1\right|}{(2 \lambda-1)^{2}}\right)\right. \\
& \left.+\frac{\left.|b|^{2}\left|6 \lambda^{2}-\frac{4}{3} \lambda^{3}-\frac{20}{3} \lambda+1\right|\right)}{8(2 \lambda-1)^{3}}\right) .
\end{aligned}
$$

Theorem 2.5. Let $f \in V(b, \lambda)$. Then

$$
\left|a_{3}-\mu a_{2}^{2}\right| \leq \frac{|b|}{2(3 \lambda-1)}\left(1+\frac{\left|(4-3 \mu) \lambda-2 \lambda^{2}-(1-\mu)\right| \cdot|b|}{2(2 \lambda-1)^{2}}\right)
$$

Proof.

$$
\begin{aligned}
a_{3}-\mu a_{2}^{2} & =\frac{b\left(2 p_{2}-p_{1}^{2}\right)}{8(3 \lambda-1)}-\frac{\left(2 \lambda^{2}-4 \lambda+1\right) a_{2}^{2}}{(3 \lambda-1)}-\mu a_{2}^{2} \\
& =\frac{b}{(3 \lambda-1)}\left(\frac{2 p_{2}-p_{1}^{2}}{8}+\frac{\left((4-3 \mu) \lambda-2 \lambda^{2}-(1-\mu)\right) b p_{1}^{2}}{16(2 \lambda-1)^{2}}\right) \\
\left|a_{3}-\mu a_{2}^{2}\right| & \leq \frac{|b|}{2(3 \lambda-1)}\left(1+\frac{\left|(4-3 \mu) \lambda-2 \lambda^{2}-(1-\mu)\right| \cdot|b|}{2(2 \lambda-1)^{2}}\right) .
\end{aligned}
$$

Theorem 2.6. Let $f \in V(b, \lambda)$. Then

$$
\left|a_{4}-a_{2} a_{3}\right| \leq \frac{|b|}{8(4 \lambda-1)}\left(31 \frac{2}{3}+\frac{4\left|7 \lambda-6 \lambda^{2}-1\right|}{3 \lambda-1}+\frac{|b|^{2} \cdot\left|8 \lambda^{4}-\frac{56}{3} \lambda^{3}+10 \lambda^{2}-\frac{44}{3} \lambda+2\right|}{4(3 \lambda-1)(2 \lambda-1)^{3}}\right) .
$$




\section{Proof.}

$$
\begin{aligned}
a_{4}-a_{2} a_{3}= & \frac{1}{4 \lambda-1}\left(\frac{b}{8}\left(4 p_{3}-4 p_{1} p_{2}+\frac{23}{24} p_{1}^{3}\right)-\left(\frac{4}{3} \lambda^{3}-6 \lambda^{2}+\frac{20}{3} \lambda-1\right) a_{2}^{3}\right) \\
& -\frac{(6 \lambda-11 \lambda+2) a_{2} a_{3}}{4 \lambda-1}-a_{2} a_{3} \\
= & \frac{b}{8(4 \lambda-1)}\left(4 p_{3}-4 p_{1} p_{2}+\frac{23}{24} p_{1}^{3}-\frac{\left(6 \lambda^{2}-7 \lambda+1\right)\left(2 p_{2}-p_{1}^{2}\right)}{3 \lambda-1}\right. \\
& \left.+\frac{\left(8 \lambda^{4}-\frac{56}{3} \lambda^{3}+10 \lambda^{2}-\frac{44}{3} \lambda+2\right) b^{2} p_{1}^{3}}{32(3 \lambda-1)(2 \lambda-1)^{3}}\right) \\
\left|a_{4}-a_{2} a_{3}\right| \leq & \frac{|b|}{8(4 \lambda-1)}\left(31 \frac{2}{3}+\frac{4\left|7 \lambda-6 \lambda^{2}-1\right|}{3 \lambda-1}+\frac{|b|^{2} \cdot\left|8 \lambda^{4}-\frac{56}{3} \lambda^{3}+10 \lambda^{2}-\frac{44}{3} \lambda+2\right|}{4(3 \lambda-1)(2 \lambda-1)^{3}}\right) .
\end{aligned}
$$

\section{References}

[1] Ş. Altinkaya and S. Yalçin, The Fekete-Szegö problem for a general class of bi-univalent functions satisfying subordinate conditions, Sahand Communications in Mathematical Analysis 5(1) (2017), 1-7.

[2] P. L. Duren, Univalent Functions, Springer-Verlag, New York, 1983.

[3] O. A. Fadipe-Joseph, A. T. Oladipo and A. U. Ezeafulukwe, Modified sigmoid function in univalent function theory, International Journal of Mathematical Sciences and Engineering Applications 7(V) (2013), 313-317.

[4] K. Kuroki and S. Owa, Notes on new class for certain analytic functions, Advances in Mathematics: Scientific Journal 1(2) (2012), 127-131.

[5] S. N. Malik, S. Mahmood, M. Raza, S. Farman, S. Zainab and N. Muhammad, Coefficient inequalities of functions associated with hyberbolic domains, Mathematics 7(1) (2019), 1-11. https://doi.org/10.3390/math7010088

[6] S. S. Miller and P. T. Mocanu, Differential Subordinations: Theory and Applications, Marcel Dekker, Inc., New York, 2000. https://doi.org/10.1201/9781482289817 